\title{
APPLICATION OF ADAPTIVE EQUALIZER FOR SIGNAL ENHANCEMENT IN TELECOMMUNICATION NETWORK
}

\author{
${ }^{1}$ OzoezeAlexanderIkechukwu, ${ }^{2}$ EgbujorEmeka Godwin, ${ }^{3}$ EzeEjike Emmanuel and \\ ${ }^{4}$ Nnaji Ugochukwu B. \\ ${ }^{1,}$ Sheda Science and Technology complex, Abuja, Nigeria. \\ ${ }^{2,}$.Enugu state University of Science and Technology, Enugu state, Nigeria. \\ ${ }^{3,}$.National Space Research and Development Agency; Centre for Basic Space Science \\ Nsukka, Enugu state, Nigeria. \\ ${ }^{4}$.Enugu state Polytechnic Iwollo, Enugu state, Nigeria.
}

This research work addressed the major concern of signal distortion in a Telecommunication networks which result in poor signal strength reception. Data communication has become increasingly in high demand in carrying out day to day activities. The need for reliable data communication-system for internet access cannot be over emphasized in networks users' still experience poor network services such as poor internet access and weak signal reception. This research work characterizes transmission networksof telecommunication systemand developed a model to equalize the phase amplitude signal of a transmission network using the Least Mean Square (LMS) adaptive equalizer algorithms. The simulation was carried out using MATLAB SIMULINK. The result obtained from the Simulations carried out in this work shows that the algorithms implemented in this research work are powerful as regards the negation of the effect of channel distortions. Thus, considering simulations carried out on both the SIMULINK model without an adaptive equalizer and that with an adaptive equalizer at the receiver, it shows that the transmission model with an adaptive equalizer enhanced the signal reception by $20 \%$. This has proved that an adaptive equalizer helps in enhancing or improving signal reception in transmission network.

Keywords: Telecommunication, Adaptive Equalizer, Signal, MATLAB/SIMULINK

\subsection{Introduction}

In some decades ago, global wireless communication has evolved from a mere convenience to a necessity one cannot afford to do without in carrying out daily life activities. The improvement in the digital signal processing, the radio transmission technologies as well as the digital computing have contributed to the introduction of a wide range of mobile wireless communication services. The global market for mobile telecommunication has rapidlyexpanded from analog telecommunication systems in the 70s and in 80s,cellular communication systems have progressed to digital cellular communication systems .In the $90 \mathrm{~s}$, the introduction of the second generation $(2 \mathrm{G})$ mobile communication system with Time Division multiple access (TDMA), Frequency Division multiple Access (FDMA), and Code Division Multiple Access (CDMA) and also the introduction of $2.5 \mathrm{G}$ such as GSM provides a reliable narrowband communication medium mostly for voice and short message service (SMS) with high mobility and high transmission rate private access WLAN such on WI-FI and Bluetooth [7]. However, due to expansion and dynamism in global market where information communication has become increasing in high demand in daily life activities. As the demand for bandwidth in telecommunication is always increasing, modern data transmission techniques are exploiting more and more physical phenomena in order to 
expand the flow of information. Thus, the third generation $(3 \mathrm{G})$ mobile networks were introduced to allow users to have access to larger amount of bandwidth that will enable them to access internet services, retrieve text messages, Image, Video and other multimedia services with a promised delivery speed of up to $2 \mathrm{Mbps}$ [2]. In recent decades, an enormous amount of work has been carried out in developing telecommunication. To fit into constraints stated in the international radio regulations, a great deal of acceleration and enhancement has been applied to former models of data encoding and modulation with increase of information density in the channel, adverse transmission effects are becoming more significant in relation to the amount of data transferred. Despite the gains of the 3G- Networks over the $2 \mathrm{G}$ Network and other analog communication systems, there still exist a number of challenges facing the 3G Networks' quality of services delivery to the end-users due signal distortion which result in poor signal reception, call drop and poor internet access which are the resultant effect of distortion in the communication channel[6].

To compensate for signal distortion of in the communication channel, present day radio equipment utilizes special procedures that involve channel analysis and digital signal processing. With vast signal processing capabilities that are available nowadays, one of the important tasks of a digital radio receiver is to adapt to environment and improve its ability to recover the already transmitted information. In carrying out this research on signal reception enhancement in a $3 \mathrm{G}$ network using an adaptive equalizer it is important to note that in $3 \mathrm{G}$ networks the base-satiation applies specific scrambling code that separates cells so as to facilitate dense frequency re-use. Inter-cell interference will appear as background noise, and the orthogonal codes may be re-used in the neighboring cells(in the same Frequency band). However, the scrambling code destroys signal cyclo-stationarity, upon the usual methods for multipath and multi-access interference (MAI) mitigation in CDMA are based [3].Thus the downlink traffic may be busty or continuous. Due to the fact that data can be received steadily, a perpetual pilot signal is multiplexed into the transmitted chip-stream.

The multiuser signal passes through a frequency and time-selective channel en-route to the receiver. Note that frequency -selective fading will occur when channel memory due to echoes causes past chips to interfere with the present chips. Thus channel memory occurs in the rich scattering environment such as in an urban environment. Frequency selectivity destroys the orthogonal nature between user codes which introduces multi-access interference (MAI) into bit estimates from the matched filter receiver. Note that the matched filter receiver which is the standard in CDMA systems is known to be affected by multi-accessinterference (MAI) [1].However, the Time-selectivity is introduced where there is relative motion between the base-station and the mobile phone or where the objects in the vicinity are in motion, thus the rate of channel variation is directly proportional to the relative velocity and the carrier frequency. More also, it is important for the receivers to adapt to changing channel conditions as quickly as possible; preferably at chip -rate [8].

\subsection{Communication System Network}

A communication system is a collection of various communication devices that allows the transmission of information from a transmitter which is the source, and passes the information to the receiver which could be one or more destinations. The transmission of the information from the transmitter to the receiver could a point-to-point or point-to-multipoints [9].

For the transmission of information to consistently perform well, each signal is always represented in a particular order of bits that should be the smallestpossible redundancy which can be achieved by the use of a source coding to perform the compression of data, and which will help also in reducing the amount of bandwidth required for information transmission [10]. However, since the transmission communication channel is time-varying; the 
transmitted signal may likely experience distortionswhich may have a modification effect on it. Error correction coding is another possible solution other than equalization in negating the effect signal distortion. Thus, error coding is an error controlling technique in information theory which is used for controlling error in data over noisy communication channel. The idea is to for the sender to encode the message being transmitted with a redundant code in form of error correction code. Examples of error correction coding are; convolution coding, block coding among others[4].

Usually, the application of the error correction coding is always done in systematic way, the extra binary digits in the series of data binary digits (bits). The receiver of the transmitted information that knows the channel coding scheme applied has the capability to remedy any error that might occur in course of transmission. After source and channel coding, if the data binary digit series to be transmitted becomes ready, it would be made suitable for the physical transmission channel. This process is known as modulation.

The data binary digit modulation is often experienced in Quadrature Amplitude Modulation. The occurring signal is thus transmitted in the communication channel. This signal ismade corrupt by factors such as signal distortion, interference and delay. At the receiver end, all operations done would find an equivalent; therefore, the received signals are demodulated and the decoded bit sequence to recover the bit sequence information originally sent. The Figure 2, according to reference [5] shows the illustration a communication system.

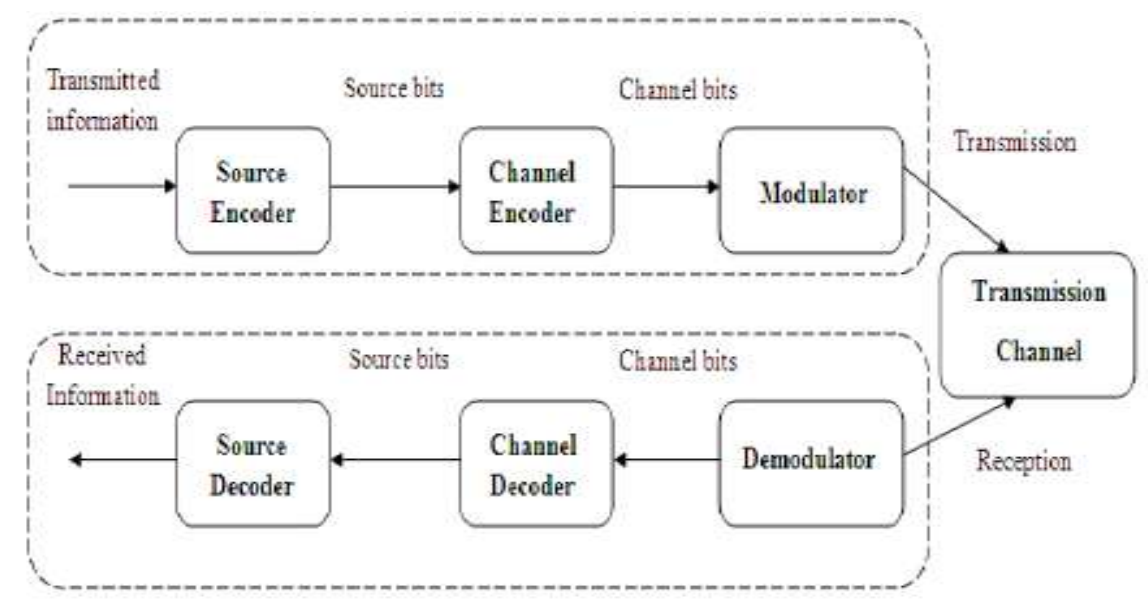

Fig. 2: Diagram of a transmission chain [5].

\section{Material and Method}

In a transmission network, signal distortion imposes a major problem in achieving increased data transmission rate with much needed accuracy. The problem associated with distortion is resolved using channel equalization with the aim of developing an equalizer whose channel impulse response and the equalizer combination is possibly nearer to $\mathrm{z}^{-} \Delta$, where $\Delta$ represents the delay in the channel. However, it is good to note that mostly the channel parameters of a transmission network are always unknown in most cases and vary with time. Thus, due to the varying nature of a system channel parameters, it is important to use an equalizer that is adaptive so as to provide an avenue whereby the characteristics of the channel would be tracked. The simulation is performed using MATLAB/SIMULINK Software. 


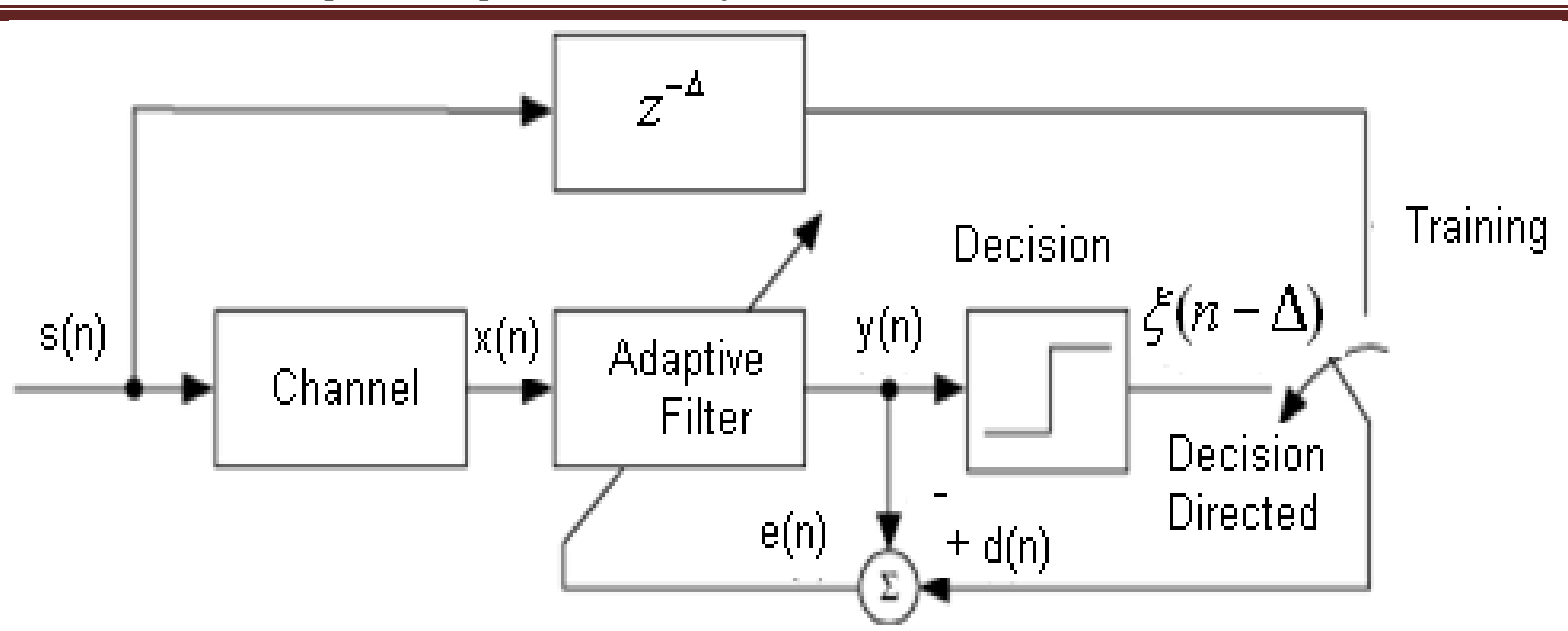

Figure 3: The diagram of a transmission system with channel equalization

From figure 3 above, we have the following;

s(n) represents the input signal transmitted through the transmission channel,

$\mathbf{x}(\mathbf{n})$ represents the output of the distorted signal from the channel without equalization.

Note that in order to reduce the effect of signal distortion, the adaptive equalization system should make use of the training mode and decision directed mode. The training mode will determine the appropriate coefficient of the adaptive filter while decision directed mode helps in the determination of the appropriate coefficients of the adaptive filter.

$\mathbf{z}^{-\Delta}$ represents the delay function while $\mathbf{d}(\mathbf{n})$ represents the delayed signal of training mode. $\mathbf{y}(\mathbf{n})$ represents the output signal of the adaptive filter, and the error signal that comes between the delayed signal and that is the error signal $\mathbf{d ( n )}$ and the output signal from the adaptive filter $\mathbf{y}(\mathbf{n})$ is represented bye(n).

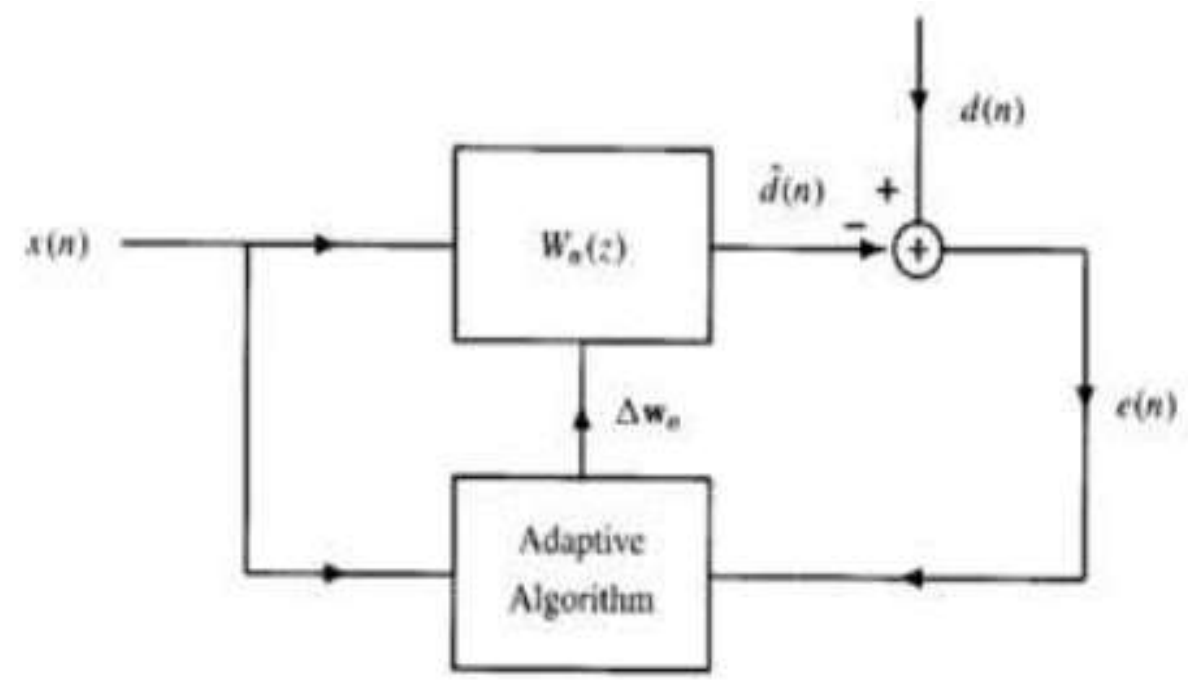

Figure3.1 Block diagram of an adaptive filter that consists of a shift- varying filter

From the above figure 3.1, we have the following;

$x(n)=$ the signal input of the desired signal $d(n)$ and signal due to interference $e(n)$

$\mathrm{d}(\mathrm{n})=$ desired signal

$\mathrm{v}(\mathrm{n})=$ unwanted signal which considered as noise

$\mathrm{e}(\mathrm{n})=$ error signal or cost function

$\mathbf{d}(\mathbf{n})=$ output signal from the variable filter or the estimated signal 
$\mathrm{w}_{\mathrm{n}}=$ variable filter

Thus:

$\mathbf{x}(n)=d(n)+\mathbf{v}(\mathbf{n})$

The equation 3.1 shows that the signal input is the combination of desired signal and the unwanted signal which is considered as noise .

The variable filter's impulse response is equal to the coefficients of the filter. However, the coefficients of a filter of order $\mathrm{p}$ are stated as

$w_{n}=\left[w_{n}(0), w_{n}(1), w_{n}(2), \ldots \ldots w_{n}(p)\right]^{T}(3.2)$

The error signal $\mathrm{e}(\mathrm{n})$ is given by the difference between the desired signal and the estimated signal, that is

$\mathbf{e}(\mathbf{n})=d(\mathbf{n})-\mathbf{d}(\mathbf{n})(3.3)$

the error signal or cost function e(n) can be obtained as the difference between the desired signal and the estimated signal output from the desired signal.

Thus, the estimation of the desired signal by the variable filter is done by convolution of the input signal with that of the impulse response

Expressing the above statement in vector notation we have, $\mathbf{d}(\mathbf{n})=\mathbf{w}_{\mathbf{n}} * \mathbf{x}(\mathbf{n})(3.4)$

Where,

$X(n)=[x(n), x(n-1), x(n-2), \ldots \ldots x(n-p)]^{T}(3.5)$

is an put signal vector.

Note that the variable filter carries out updates of the filter coefficients at every time interval, and that is represented as $\mathbf{w}_{\mathbf{n}+\mathbf{1}}=\mathbf{w}_{\mathbf{n}}+\mathbf{w}_{\mathbf{n}}+\Delta \mathbf{w}_{\mathbf{n}}(\mathbf{3 . 6})$

Note also that from equation 3.6, the correction factor of the filter coefficients given as $\Delta \mathbf{w}_{\mathbf{n} .}$,thus, the equalizer's adaptive algorithm generates the correction factor considering both the signal input $\mathbf{x}(\mathbf{n})$ and error signal $\mathbf{e}(\mathbf{n})$

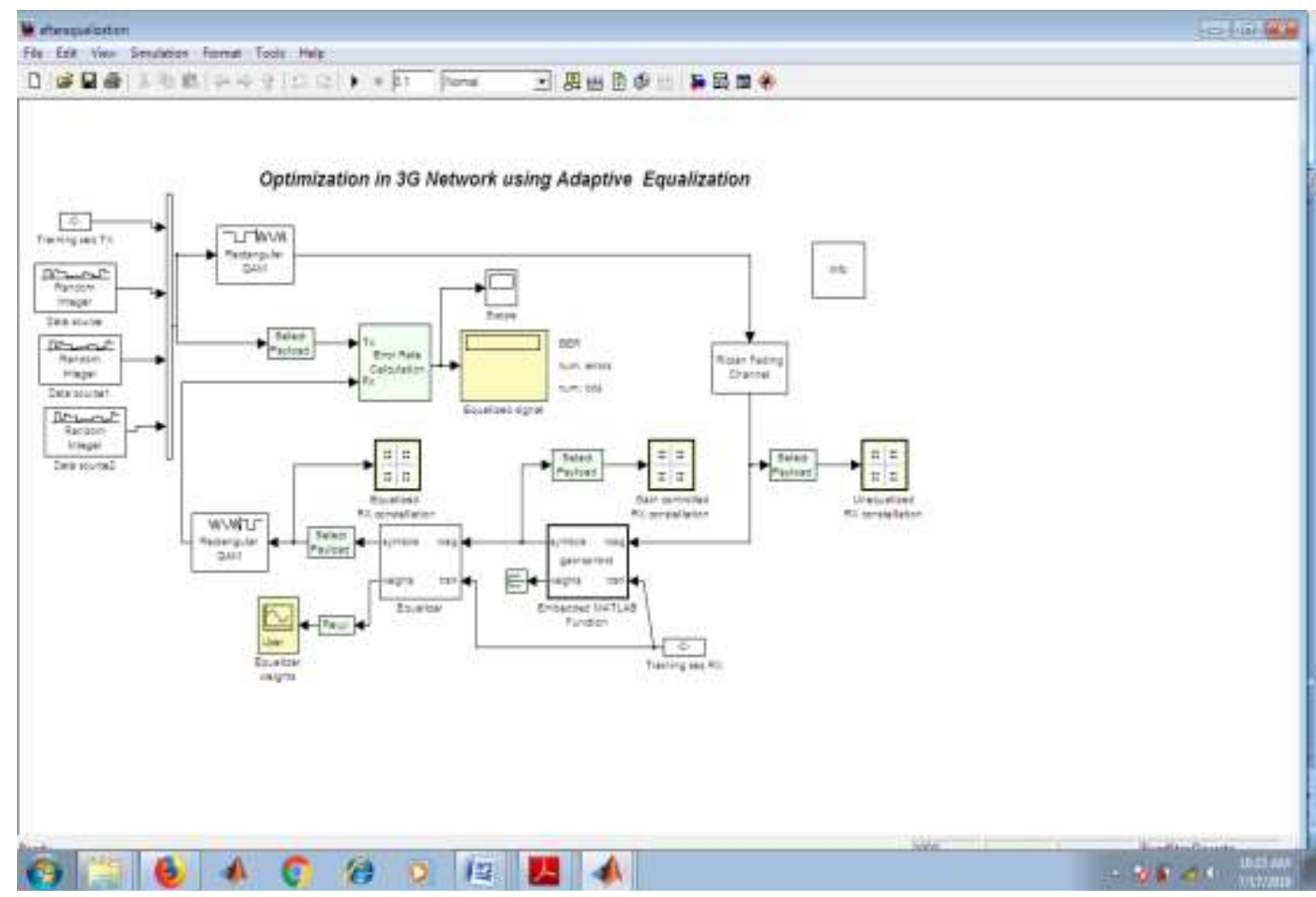

Figure 3.2: Simulation of Adaptive Equalization 


\section{1: The Program Flow Chart}

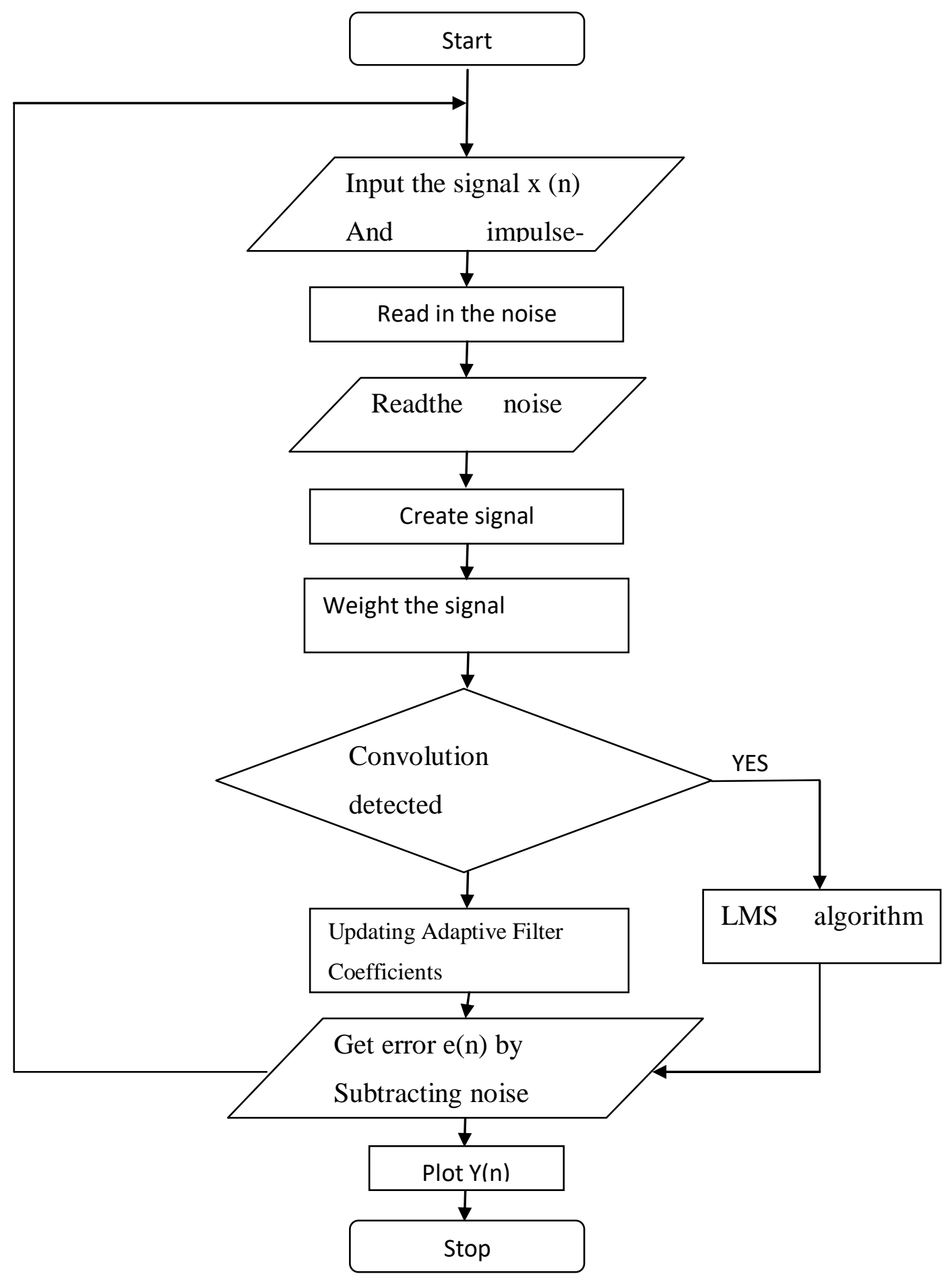

\subsection{Result and Discussion}

In figure 4.1, this correction allows us to compute the transmitter-receiver distance from measured RSSI values. The measured values follow the progression of the function graphs. In figure 4.1, it is observed that the distance from the transmitter antenna to the receiver antenna affects the signal power received at the receiver antenna. As shown in Figure 4.1, the received signal strength varies continuously with the change in locations with varying distance. That is to say that changes in locations lead to the variation in RSSI with a power = 
$46 \mathrm{dBm}$. At the distance above $700 \mathrm{~m}$, the RSSI weakened and the signal reception in transmission network became poor. For short and midrange distances the expected significant degradation of the resulting RSSI values could not be observed. However, distances beyond 700 m suddenly produced $100 \%$ packet loss rate.

The adaptive equalizer plays an important role in the result obtained. In figure 4.2 , it is observed that the location affects the power received. The received signal strength varies continuously with the change of location. That is to say, the changes in location lead to variation for RSSI with a power $=46 \mathrm{dBm}$. With the adaptive equalizer, it was noticed that the RSSI increased across the varied location. At the distance above $700 \mathrm{~m}$, the RSSI was still strong and the signal reception in transmission network was strong. For short, midrange and long distances the expected significant degradation of the resulting RSSI values could not be observed significantly.

The Comparison of measured and simulated values of RSSI Variations with changes in distance for MS transmission power $=46 \mathrm{dBm}$ with and without adaptive equalizer as shown in figure 4.3, the strength of the received-signal varies continuously with the change of location. That is to say, the changes in location lead to the variation for RSSI with a power = $46 \mathrm{dBm}$. At the distance above $700 \mathrm{~m}$ for model without adaptive equalizer, the RSSI weakened and the signal reception in transmission network became poor. But the adaptive equalizer played an important role in the result obtained. In using an adaptive equalizer, it was noticed that the RSSI improved across the various distances. At the distance above $700 \mathrm{~m}$, the RSSI was still strong and the signal reception in transmission network was strong. For short- range, mid-range and long-range distances, the expected significant degradation of the resulting RSSI values was not significantly noticed.

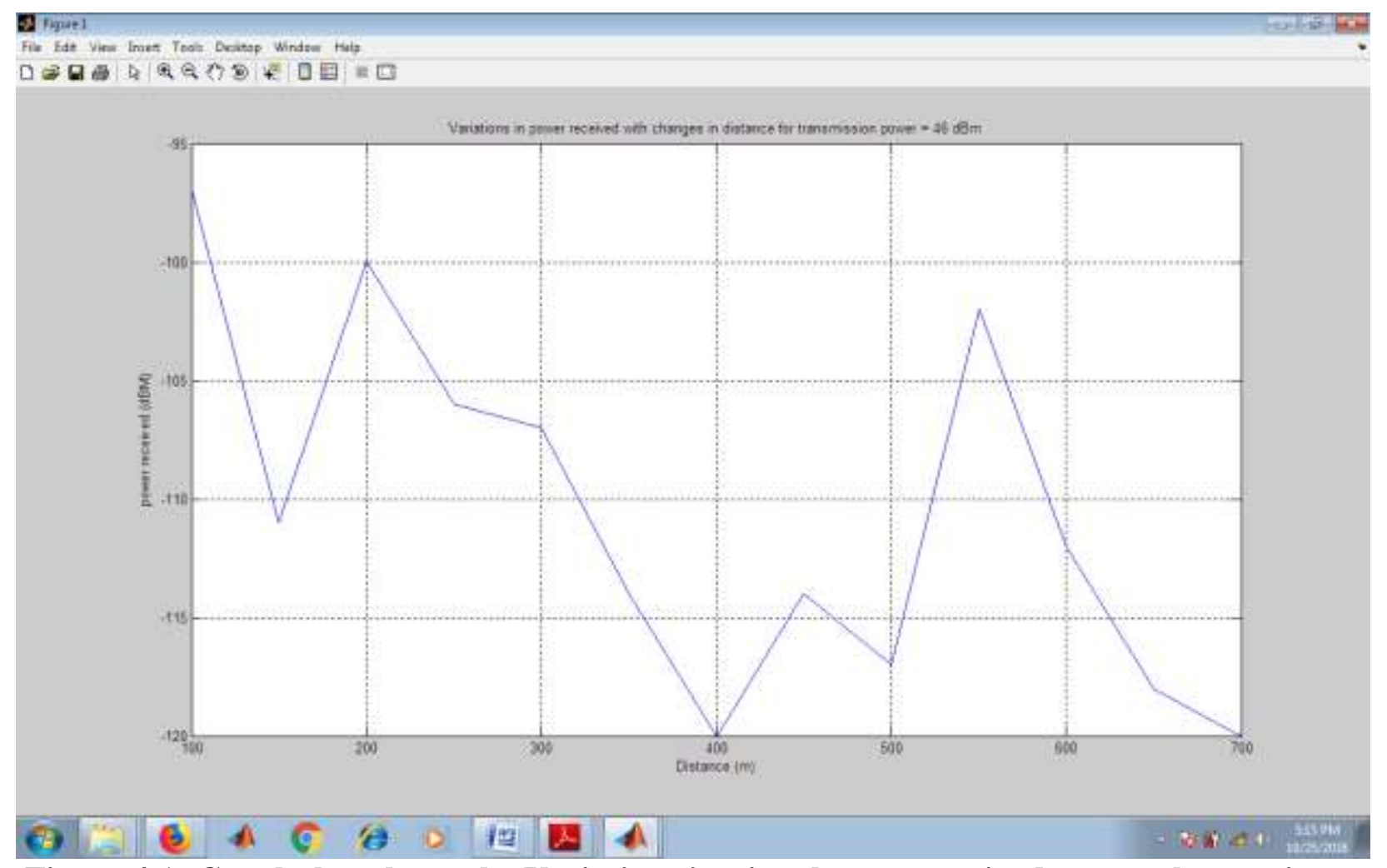

Figure 4.1: Graph that shows the Variations in signalpower received versus changes in distance for MS transmission power $=46 \mathrm{dBm}$ without adaptive equalizer. 


\section{Table 4.1: Power received (With Adaptive Equalizer)}

\begin{tabular}{|c|l|}
\hline Distance $(\mathbf{m})$ & \\
\hline 100 & -77 \\
\hline 150 & -91 \\
\hline 200 & -80 \\
\hline 250 & -86 \\
\hline 300 & -87 \\
\hline 350 & -94 \\
\hline 400 & -100 \\
\hline 450 & -94 \\
\hline 500 & -97 \\
\hline 550 & -82 \\
\hline 600 & -92 \\
\hline 650 & -98 \\
\hline 700 & -100 \\
\hline &
\end{tabular}

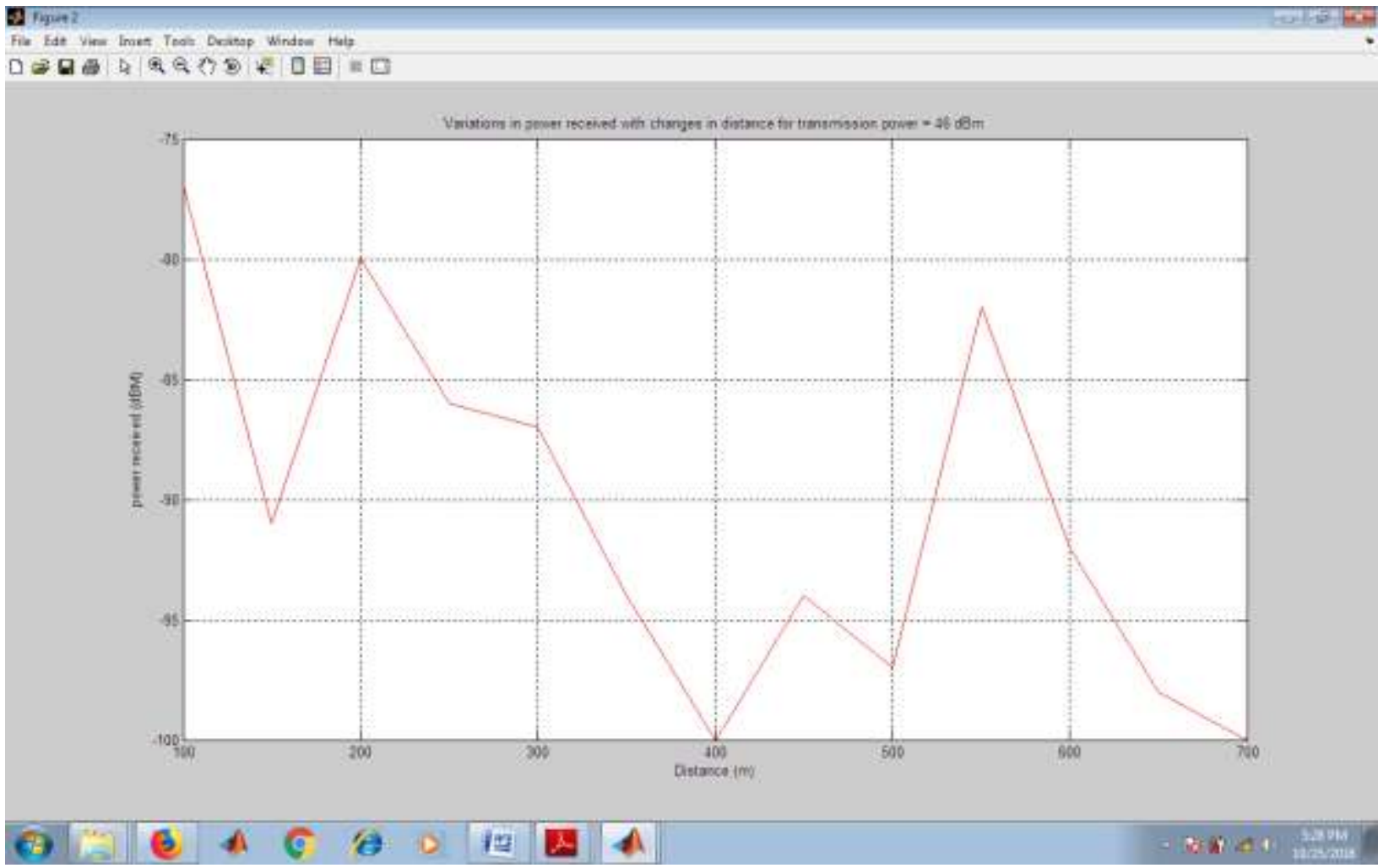

Figure 4.2: Graph that shows the Variations in Signal power received versus changes in distance for MS transmission power $=46 \mathrm{dBm}$ with adaptive equalizer.

Table 4.2: Comparison ofPower received.

\begin{tabular}{|c|l|l|}
\hline Distance (m) & \multicolumn{1}{|c|}{$\begin{array}{c}\text { RSSI (dBm) Without } \\
\text { Adaptive Equalizer }\end{array}$} & $\begin{array}{c}\text { RSSI (dBm) With Adaptive } \\
\text { Equalizer }\end{array}$ \\
\hline 100 & -97 & -77 \\
\hline 150 & -111 & -91 \\
\hline 200 & -100 & -80 \\
\hline 250 & -106 & -86 \\
\hline
\end{tabular}




\begin{tabular}{|l|l|l|}
\hline \hline 300 & -107 & -87 \\
\hline 350 & -114 & -94 \\
\hline 400 & -120 & -100 \\
\hline 450 & -114 & -94 \\
\hline 500 & -117 & -97 \\
\hline 550 & -102 & -82 \\
\hline 600 & -112 & -92 \\
\hline 650 & -118 & -98 \\
\hline 700 & -120 & -100 \\
\hline
\end{tabular}

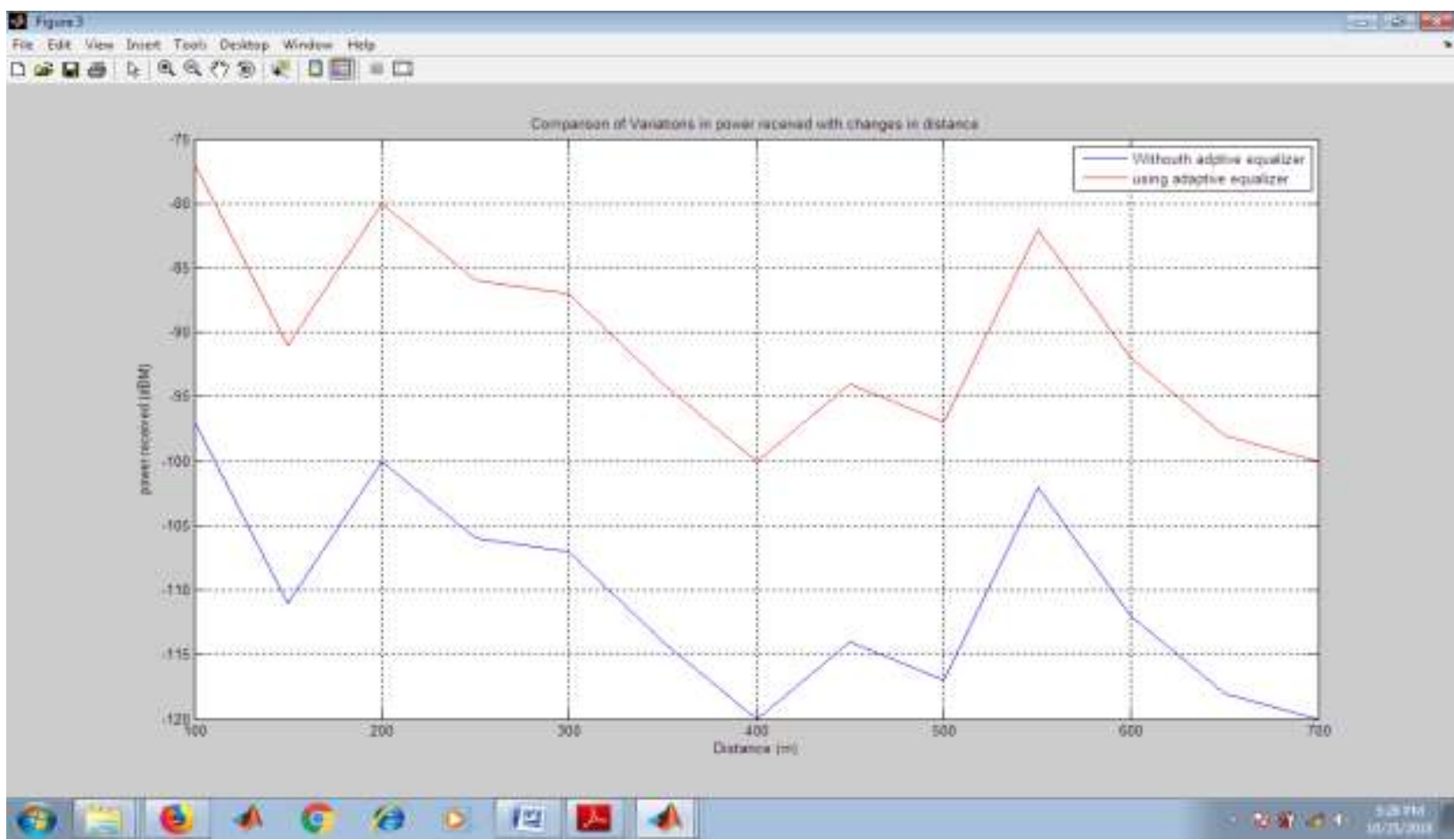

Figure 4.3: Comparison of measured and simulated values of RSSI variations with changes in distance for MS transmission power $=46 \mathrm{~dB}$, with and without adaptive equalizer

\section{Conclusion}

This research work summarizes the basic theoretical channel propagation model for radio signals in a free space environment. We also explained our corresponding real-time test-bed in detail before presenting and analyzing the Reception Signal Strength Index measurement series. By choosing suitable parameters, the outdoor experiments closely follow the theoretical free space propagation model with ground reflection.

Simulations have shown that the algorithm used in this project is robust with respect to the channel distortions. For a short-range, mid-range and long-range distances, the expected significant degradation of the resulting RSSI values could not be significantly noticed . Thus; this has shown that an adaptive equalizer helps to enhance signal reception in transmission network. 


\section{Reference}

[1]Gregory E. B.(2013). "Optimizing the rake receiver for the CDMA downlink", Proc. IEEE Vehicular Technology Conference, pp. 742-745.

[2]Mary, B. (2010). Selling the cell phones - history of cellular phones.

[3]Ruxandra, L., and Sergio, V. (2009). "Linear multiuser detectors for synchronouscodedivision multiple-access channels", IEEE Trans. on Information Theory, vol. 35, no. 1, pp. 123-135.

[4]Samina C., and Zoltowski, M.D., (2012). "Adaptive MMSE equalization for wideband CDMA forward link with time-varying frequency selective channels", Proc. IEEE Internat. Conf. on Acoustics, Speech, and Signal Processing, vol. 3, pp. 2605-2608.

[5]Vasundhara, A.M., Sravanthi, A.G., (2014). ' Study of Median Filter in DifferentNoise Density Environments in Binary Images', ISSN (Online) 2278- 5841,ISSN (Print) 2320- 5156, International Journal of Research in Computer and Communication Technology, Vol 3, Issue 6.

[6]Werner, S., and Lilleberg, J., (2009). "Downlink channel decorrelation in CDMA systems with long codes", Proc. IEEE Vehicular Technology Conference, pp. 16141617.Communications: Algorithms, Performance, and Architecture, Journal of VLSI Signal Processing, vol. 30, no. 1, pp. 273-291.

[7]Wornell, G.W.,(1996). "Spread-response precoding for communication over fading channels,"IEEE Transactions on information theory, pp. 488-501.

[8]Zhou, W., Xie, B.,Jun-de, S., (2007). "Link level simulation and performanceestimation of WIMAX IEEE 802.16e", in 2nd Int. Conf. Pervasive Computing and Applications, pp.667-671.

[9]Zoltowski, M.D., and Thomas P. K., (2009). "Two-channel zero forcing equalization on CDMA forward link: Trade-offs between multi-user access interference and diversity gains", Proc. Asilomar Conf. on Signals, Systems and Computers, vol. 2, pp. 15411545.

[10]Zoltowski, M.D., and Thomas P. K., (2009). "Two-channel zero forcing equalization on CDMA forward link: Trade-offs between multi-user access interference and diversity gains", Proc. Asilomar Conf. on Signals, Systems and Computers, vol. 2, pp. 15411545. 